\title{
Pitfalls associated with evaluating enzymatic quorum quenching activity: the case of MomL and its effect on Pseudomonas aeruginosa and Acinetobacter baumannii biofilms
}

\author{
Yunhui Zhang ${ }^{1}{ }^{\text {, Gilles Brackman }}{ }^{1}$, Tom Coenye ${ }^{\text {Corresp. } 1}$ \\ ${ }^{1}$ Ghent University \\ Corresponding Author: Tom Coenye \\ Email address: Tom.Coenye@UGent.be
}

Background. The enzymatic degradation of quorums sensing (QS) molecules (called quorum quenching, QQ) has been considered as a promising anti-virulence therapy to treat biofilm-related infections and combat antibiotic resistance. The recently-discovered QQ enzyme MomL has been reported to efficiently degrade different $N$-acyl homoserine lactones (AHLs) of various Gram-negative pathogens. Here we investigated the effect of MomL on biofilms formed by two important nosocomial pathogens, Pseudomonas aeruginosa and Acinetobacter baumannii. Methods. MomL was expressed in E.coli BL21 and purified. The activity of MomL on AHLs with hydroxyl substituent was tested. Biofilms of $P$. aeruginosa PAO1 and Acinetobacter strains were formed in 96-well microtiter plates. Biofilm formation was evaluated by crystal violet staining, plating and fluorescence microscopy. The effect of MomL on biofilm susceptibility to antibiotics was also tested. We further evaluated MomL in dual-species biofilms formed by $P$. aeruginosa and $A$.

baumannii, and in biofilms formed in a wound model. The effect of MomL on virulence of $A$. baumannii was also tested in the Caenorhabditis elegans model. Results. MomL reduced biofilm formation and increased biofilm susceptibility to different antibiotics in biofilms of P. aeruginosa PAO1 and A. baumannii LMG 10531 formed in microtiter plates in vitro. However, no significant differences were detected in the dual-species biofilm and in wound model biofilms. In addition, MomL did not affect virulence of $A$. baumannii in the $C$. elegans model. Finally, the effect of MomL on biofilm of Acinetobacter strains seems to be straindependent. Discussion. Our results indicate that although MomL showed a promising anti-biofilm effect against $P$. aeruginosa and $A$. baumanii biofilms formed in microtiter plates, the effect on biofilm formation under conditions more likely to mimic the real-life situation was much less pronounced or even absent. Our data indicate that in order to obtain a better picture of potential applicability of QQ enzymes for the treatment of biofilm-related infections, more elaborate model systems need to be used. 
1 Pitfalls associated with evaluating enzymatic quorum quenching activity: the

2 case of MomL and its effect on Pseudomonas aeruginosa and Acinetobacter

3 baumannii biofilms

4

5 Yunhui Zhang ${ }^{1}$, Gilles Brackman ${ }^{1}$ and Tom Coenye ${ }^{1^{*}}$

6

$7{ }^{1}$ Laboratory of Pharmaceutical Microbiology, Ghent University, Gent, Belgium.

8

9

10 Corresponding Author: Tom Coenye ${ }^{1}$

11 Email address: tom.coenye@Ugent.be 


\section{Abstract}

13 Background. The enzymatic degradation of quorums sensing (QS) molecules (called quorum quenching, QQ) has been considered as a promising anti-virulence therapy to treat biofilmrelated infections and combat antibiotic resistance. The recently-discovered QQ enzyme MomL has been reported to efficiently degrade different $N$-acyl homoserine lactones (AHLs) of various Gram-negative pathogens. Here we investigated the effect of MomL on biofilms formed by two important nosocomial pathogens, Pseudomonas aeruginosa and Acinetobacter baumannii.

Methods. MomL was expressed in E.coli BL21 and purified. The activity of MomL on AHLs with hydroxyl substituent was tested. Biofilms of $P$. aeruginosa PAO1 and Acinetobacter strains were formed in 96-well microtiter plates. Biofilm formation was evaluated by crystal violet staining, plating and fluorescence microscopy. The effect of MomL on biofilm susceptibility to antibiotics was also tested. We further evaluated MomL in dual-species biofilms formed by $P$. aeruginosa and A. baumannii, and in biofilms formed in a wound model. The effect of MomL on virulence of $A$. baumannii was also tested in the Caenorhabditis elegans model.

Results. MomL reduced biofilm formation and increased biofilm susceptibility to different antibiotics in biofilms of $P$. aeruginosa PAO1 and A. baumannii LMG 10531 formed in microtiter plates in vitro. However, no significant differences were detected in the dual-species biofilm and in wound model biofilms. In addition, MomL did not affect virulence of $A$. baumannii in the $C$. elegans model. Finally, the effect of MomL on biofilm of Acinetobacter strains seems to be strain-dependent.

Discussion. Our results indicate that although MomL showed a promising anti-biofilm effect against $P$. aeruginosa and $A$. baumanii biofilms formed in microtiter plates, the effect on biofilm formation under conditions more likely to mimic the real-life situation was much less pronounced or even absent. Our data indicate that in order to obtain a better picture of potential applicability of QQ enzymes for the treatment of biofilm-related infections, more elaborate model systems need to be used. 
40

41

42

\section{Introduction}

Quorum sensing (QS) is a widespread communication process that allows bacteria to coordinate their group behavior based on the production, detection and response to extracellular signal molecules (Bassler \& Losick 2006; Williams et al. 2007). QS regulates gene expression related to biofilm formation, motility and production of virulence factors in many Gram-negative and Gram-positive pathogens, and interfering with QS has been intensively studied as a promising anti-virulence therapy to combat bacterial infections and antibiotic resistance (Hentzer \& Givskov 2003; LaSarre \& Federle 2013; Rutherford \& Bassler 2012). Many natural and synthetic compounds have been found to inhibit QS, and several quorum quenching (QQ) enzymes mainly targeting $N$-acyl homoserine lactone (AHL) based QS in Gram-negative bacteria have been described as well (Brackman \& Coenye 2015; Fetzner 2015; Rasmussen \& Givskov 2006; Tang \& Zhang 2014). Some of these QS inhibitors (QSIs) and QQ enzymes have shown promising anti-virulence effects both in vitro and in vivo. For instance, furanones which resemble AHLs and are able to bind to QS receptors have been reported to reduce biofilm formation and enhance bacterial clearance in Pseudomonas aeruginosa lung infection in mice (Hentzer et al. 2002; Wu et al. 2004). Baicalin hydrate and cinnamaldehyde (QSIs targeting AHL-based QS in $P$. aeruginosa and Burkholderia cepacia complex) as well as hamamelitannin (a QSI targeting the peptide-based QS system present in Staphylococcus aureus) increase biofilm susceptibility to antibiotics and survival of infected Galleria mellonella larvae and Caenorhabditis elegans, as well as decrease the microbial load in a mouse pulmonary infection model (Brackman et al. 2011). As for QQ enzymes, an AiiM-producing P. aeruginosa mutant showed reduced lung injury and increased survival in an in vivo study on mice with pneumonia (Migiyama et al. 2013), and an inhaled lactonase SsoPox-I was also reported to reduce virulence of P. aeruginosa and mortality in rat pneumonia (Hraiech et al. 2014).

Previously MomL, a novel AHL lactonase belonging to the metallo- $\beta$-lactamase superfamily was identified and characterized (Tang et al. 2015). It has high degrading activities towards shortand long-chain AHLs with or without an oxo-group at the C-3 position (Tang et al. 2015). MomL can reduce pyocyanin and protease production by $P$. aeruginosa and attenuated the virulence of $P$. aeruginosa in a $C$. elegans infection model (Tang et al. 2015), but its effect on biofilm formation of $P$. aeruginosa and other Gram-negative pathogens was not tested yet. 
Besides $P$. aeruginosa, Acinetobacter baumannii has also been recognized as an increasingly prevalent Gram-negative opportunistic pathogen responsible for severe nosocomial infections (Gonzalez-Villoria \& Valverde-Garduno 2016; Peleg et al. 2008). Resistance of P. aeruginosa and A. baumannii strains to multiple antibiotic classes complicates the treatment for these infections and poses considerable therapeutic challenges worldwide (Potron et al. 2015). One of the main factors contributing to their reduced antibiotic susceptibility and to treatment failure is biofilm formation both on tissues and abiotic surfaces (Donlan \& Costerton 2002; Hall-Stoodley et al. 2004; Longo et al. 2014). Biofilms of both P. aeruginosa and A. baumannii are known to be regulated by AHL-based QS. In P. aeruginosa, $N$-(3-oxododecanoyl)-L-homoserine lactone (3-oxo-C12-HSL) and N-butyryl-L-homoserine lactone (C4-HSL) are used by the Las and Rhl QS system, respectively (Pesci et al. 1997), and these AHLs can both be degraded by MomL (Tang et al. 2015). One AHL synthase belonging to LuxI family, AbaI, has been reported to catalyze the synthesis of $\mathrm{N}$-(3-hydroxydodecanoyl)-L-HSL (3-OH-C12-HSL) in Acinetobacter nosocomialis M2 (Niu et al. 2008), but other AHLs with varied chain lengths and substituents are also found in Acinetobacter strains (Bhargava et al. 2010; González et al. 2009). The biofilmforming ability of an abaI mutant was reduced by around $40 \%$ compared to the corresponding wildtype strain (Niu et al. 2008). Compared to the extensive literature on inhibiting QS pathways and virulence in P. aeruginosa (Aybey \& Demirkan 2016; Furiga et al. 2016; Hentzer et al. 2003; O’Loughlin et al. 2013; Yin et al. 2015), there are fewer reports on inhibiting QS and biofilm formation in A. baumannii (Bhargava et al. 2015; Chow et al. 2014; Saroj \& Rather 2013).

In the present study, we tested the anti-biofilm activity of MomL against $P$. aeruginosa and $A$. baumannii, and further evaluated the effect of MomL under more complex conditions such as in dual-species biofilm and in a wound model system with the aim to obtain a better knowledge base regarding the possible development of QQ enzymes as anti-virulence therapy.

\section{Material \& Methods}

\section{Bacterial strains, culture conditions and chemicals}

P. aeruginosa PAO1, A. calcoaceticus LMG 10517, A. nosocomialis M2 and A. baumannii LMG 10520, LMG 10531 and AB5075 were cultured on tryptic soy agar (TSA) or in Mueller-Hinton 
99

100

101

102

103

104

105

106

107

108

109

110

111

112

113

114

115

116

117

118

119

120

121

122

123

124

125

126

broth $(\mathrm{MH})$ at $37^{\circ} \mathrm{C}$. Escherichia coli BL21(DE3) harboring MomL expression plasmid pET24a(+)-momL-(-SP) (Tang et al. 2015) was cultured on Luria-Bertani (LB) agar supplemented with kanamycin $(50 \mu \mathrm{g} / \mathrm{mL})$ at $37^{\circ} \mathrm{C}$. The AHL biosensor Agrobacterium tumefaciens A136 (pCF218) (pCF372) (Zhu et al. 1998) was maintained on LB agar supplemented with spectinomycin $(50 \mu \mathrm{g} / \mathrm{mL})$ and tetracycline $(4.5 \mu \mathrm{g} / \mathrm{mL})$, and grown in AT minimal medium (Tempé et al. 1977) containing 0.5\% (wt/vol) glucose for detecting AHLs in the liquid X-Gal (5-bromo-4-chloro-3-indolyl- $\beta$-D-galactopyranoside) assay. 3-OH-C12-HSL was purchased from Sigma-Aldrich and dissolved in dimethyl sulfoxide (DMSO) as stock solution (100 mM). Caenorhabditis elegans N2 (glp-4; sek-1) was propagated under standard conditions, synchronized by hypochlorite bleaching, and cultured on nematode growth medium using E. coli OP50 as a food source (Stiernagle 1999).

\section{MomL expression and purification}

MomL was expressed and purified according to Tang et al., 2015. In brief, protein expression was induced by $0.5 \mathrm{mM}$ IPTG (isopropyl- $\beta$-D-thiogalactopyranoside) when $E$. coli cells in LB reaching an optical density at $600 \mathrm{~nm}(\mathrm{OD} 600)$ of 0.5 to 0.7 . The induction was carried out at $16^{\circ} \mathrm{C}$ with moderate shaking $(150 \mathrm{rpm})$ for $12 \mathrm{~h}$. Cells were harvested and sonicated, and the obtained supernatant was loaded on NTA-Ni (Qiagen) columns for purification according to the manufacturer's instruction. Desalting of the protein solution was accomplished by Amicon Ultra15 centrifugal filter devices, and the purified MomL was stored at $-20^{\circ} \mathrm{C}$ in Tris- $\mathrm{HCl}$ buffer (50mM, pH 6.5) with $25 \%$ glycerol.

\section{Detection for degradation of 3-OH-C12-HSL}

The amount of 3-OH-C12-HSL was quantified using A. tumefaciens A136 liquid X-gal assay and expressed as the normalized $\beta$-galactosidase activity as previously described (Tang et al. 2013). The correction factor $\mathrm{a}$ and $\mathrm{b}$ were obtained and calculated for our experimental conditions, and the final formula to calculate the normalized $\beta$-galactosidase activity is $\frac{0.716 \times O D 492-O D 620}{0.205 \times O D 620-O D 492}$. To test the degradation of 3-OH-C12-HSL by MomL, 3-OH-C12-HSL $(10 \mu \mathrm{M})$ was mixed with 
127 MomL in different concentrations $(0.05-5 \mu \mathrm{g} / \mathrm{mL})$ and incubated at $37^{\circ} \mathrm{C}$ for $1 \mathrm{~h}$. No MomL was

128 added to the control. Afterwards the residual 3-OH-C12-HSL was quantified by adding $10 \mu \mathrm{L}$

129 solution to the A136 biosensor, as described previously (Tang et al. 2013).

\section{Biofilm formation assays}

132

133

134

135

136

137

138

139

140

141

142

143

144

145

146

147

148

149

150

151

152

153

154

Overnight cultures of $P$. aeruginosa and Acinetobacter strains in $\mathrm{MH}$ broth were diluted to contain approximately $5 \times 10^{7} \mathrm{CFU} / \mathrm{mL} .90 \mu \mathrm{L}$ of this diluted bacterial suspension was transferred to the wells of a round-bottomed 96-well microtiter plate. Uninoculated $\mathrm{MH}$ broth served as blank control. To test the effect of MomL on biofilms, $10 \mu \mathrm{L}$ purified enzyme (in different concentrations) was added to the wells, while $10 \mu \mathrm{L}$ Tris- $\mathrm{HCl}$ buffer (50mM, pH 6.5) with 25\% glycerol was added to the control. The plate was incubated at $37^{\circ} \mathrm{C}$ for 4 hours before the supernatant was removed. The wells were rinsed once with sterile physiological saline (PS) and re-filled with fresh medium $(90 \mu \mathrm{L})$ and $\operatorname{MomL}(10 \mu \mathrm{L})$. The plate was incubated at $37^{\circ} \mathrm{C}$ for an additional 20 hours. The biofilm biomass was quantified by crystal violet (CV) staining as described previously (Peeters et al. 2008). After rinsing the wells with sterile PS, the biofilm was fixed with $100 \mu \mathrm{L} 99 \%$ methanol for $15 \mathrm{~min}$ and stained with $100 \mu \mathrm{L} 0.1 \% \mathrm{CV}$ for $20 \mathrm{~min}$. The excess $\mathrm{CV}$ was removed by washing the plates under running tap water and bound $\mathrm{CV}$ was released by adding $150 \mu \mathrm{l}$ of $33 \%$ acetic acid. The absorbance was measured at $590 \mathrm{~nm}$.

\section{Biofilm susceptibility assays}

After a 24 h-biofilm of $P$. aeruginosa or A. baumannii strains was formed as described above either in presence of MomL or not, the plate was emptied and biofilm cells were rinsed with sterile PS. Antibiotics were dissolved in PS and $90 \mu \mathrm{L}$ of these solutions were added to treat the biofilm for another $24 \mathrm{~h}$, either with or without $10 \mu \mathrm{l} \mathrm{MomL}$. Tobramycin (TOB; $4 \mu \mathrm{g} / \mathrm{mL}$ as final concentration), ciprofloxacin (CIP; $0.5 \mu \mathrm{g} / \mathrm{mL}$ ), meropenem (MEM; $16 \mu \mathrm{g} / \mathrm{mL}$ ) and colistin $(\mathrm{CST} ; 16 \mu \mathrm{g} / \mathrm{mL})$ were used to treat the biofilm of $P$. aeruginosa PAO1; TOB $(6 \mu \mathrm{g} / \mathrm{mL}), \mathrm{CIP}(4$ $\mu \mathrm{g} / \mathrm{mL}), \operatorname{MEM}(8 \mu \mathrm{g} / \mathrm{mL})$ and CST $(16 \mu \mathrm{g} / \mathrm{mL})$ were used to treat the biofilm of the $A$. baumannii strains. The supernatant was removed and the wells were washed once with sterile 
155 PS. To release bacterial cells from biofilm, two cycles of vortex (5 mins) and sonication (5 mins)

156 were performed, and the number of CFU/biofilm was determined by plating the resulting

157 suspensions on TSA.

158

159

\section{Fluorescence microscopy}

160

161

162

163

164

165

166

167

168

169

170

171

172

173

174

175

176

177

178

179

180

181

Biofilms of $P$. aeruginosa PAO1 or A. baumannii strains were formed in the absence or presence of MomL and treated with antibiotics as described above using a flat-bottomed 96-well microtiter plates. $3 \mu \mathrm{L}$ SYTO9 and $3 \mu \mathrm{L}$ propidium iodide were diluted to $1 \mathrm{~mL}$ in sterile PS, and $100 \mu \mathrm{L}$ of this staining solution was transferred to each well. The plate was incubated for $15 \mathrm{~min}$ at room temperature and fluorescence microscopy was performed with EVOS FL Auto Imaging System (Life Technologies). The red fluorescent signal was detected with 531/40 nm excitation filter cube and 593/40 $\mathrm{nm}$ emission filter cube and the green fluorescent signal was detected with 470/22 nm excitation filter cube and 510/42 nm emission filter cube.

\section{Dual-species biofilm formation}

Overnight cultures of $P$. aeruginosa and A. baumannii strains in $\mathrm{MH}$ broth were diluted to contain approximately $5 \times 10^{5} \mathrm{CFU} / \mathrm{mL}$ and $5 \times 10^{7} \mathrm{CFU} / \mathrm{mL}$, respectively, and equal volume of suspensions of $P$. aeruginosa and A. baumannii were mixed. MomL $(200 \mu \mathrm{g} / \mathrm{mL})$ and tobramycin $(6 \mu \mathrm{g} / \mathrm{mL})$ were added as described above. To quantify CFU in the dual-species biofilm, Pseudomonas Isolation Agar (Difco) and TSA supplemented with $5 \mu \mathrm{g} / \mathrm{mL}$ cefsulodin were used as selective media for $P$. aeruginosa and $A$. baumannii respectively.

\section{Biofilm formation in wound model}

Artificial dermis composed of hyaluronic acid and collagen was used in our wound model, as described before (Brackman et al. 2016). Each disk of artificial dermis was placed in 24-well microtiter plate. One $\mathrm{mL}$ medium containing Bolton Broth, heparinized bovine plasma and freeze-thaw laked horse blood cells was added on and around the dermis. Suspensions $(10 \mu \mathrm{L})$ of 
182 P. aeruginosa or $A$. baumannii containing $10^{4}$ bacterial cells were added on the top of dermis.

183 Final concentrations of MomL added were $200 \mu \mathrm{g} / \mathrm{mL}$ and $10 \mu \mathrm{g} / \mathrm{mL}$ for $P$. aeruginosa and $A$. 184 baumannii, respectively. Tobramycin $(10 \mu \mathrm{g} / \mathrm{mL})$ was added after $8 \mathrm{~h}$ incubation at $37^{\circ} \mathrm{C}$. After 185 24h, the infected dermis was washed with $1 \mathrm{~mL}$ PS and was transferred into $10 \mathrm{ml} \mathrm{PS}$. Biofilm 186 cells on the dermis were loosen and collected by three cycles of vortex (30 s) and sonication (30 $187 \mathrm{~s})$. The number of CFU/dermis was quantified by standard plating techniques.

\section{C. elegans survival assay}

190

191

192

193

194

195

196

197

198

199

200

201

202

203

204

205

206

207

The C. elegans survival assay was carried out as described before with minor modification (Brackman et al. 2011). Synchronized worms (L4 stage) were suspended in medium containing $95 \% \mathrm{M} 9$ buffer $\left(3 \mathrm{~g}\right.$ of $\mathrm{KH}_{2} \mathrm{PO}_{4}, 6 \mathrm{~g}$ of $\mathrm{Na}_{2} \mathrm{HPO}_{4}, 5 \mathrm{~g}$ of $\mathrm{NaCl}$, and $1 \mathrm{ml}$ of $1 \mathrm{M} \mathrm{MgSO}{ }_{4} \cdot 7 \mathrm{H}_{2} \mathrm{O}$ in 1 liter of water) and 5\% brain heart infusion broth (Oxoid), and $25 \mu \mathrm{L}$ of this nematode suspension was transferred to the wells of a 96-well microtiter plate. Overnight culture of $A$. baumannii was suspended in the assay medium and added in a final concentration of $2.5 \times 10^{7}$ $\mathrm{CFU} / \mathrm{ml}$. MomL was added in a final concentration of $10 \mu \mathrm{g} / \mathrm{mL}$. The plates were incubated at $25^{\circ} \mathrm{C}$ for $24 \mathrm{~h}$. The fraction of dead worms was determined by counting the number of dead worms and the total number of worms in each well.

\section{Statistics}

The normal distribution of the data was checked by the D'Agostino-Pearson normality test. Normally distributed data were analyzed by one-way ANOVA, and non-normally distributed data were analyzed by the Kruskal-Wallis test or the Mann-Whitney test. All statistical analyses were carried out using GraphPad Prism 6.0.

\section{Results}

Degradation of 3-OH-C12-HSL by purified MomL 
208 MomL was produced in E. coli and subsequently successfully purified (Fig. 1). Although MomL

209 had been shown to degrade various AHLs (Tang et al. 2015), its activity on AHLs with hydroxyl

210 substituent at the C3 position was not tested yet. We could demonstrate that MomL, in a

211 concentration of $1 \mu \mathrm{g} / \mathrm{mL}$ or higher, can degrade almost all 3-OH-C12-HSLs $(10 \mu \mathrm{M})$ under the

212 experimental conditions used in the present study (Fig. 2).

\section{Effect of MomL on biofilm formation by $P$. aeruginosa and $A$. baumannii}

215

216

217

218

219

220

221

222

223

224

225

226

227

228

229

230

231

232

233

234

235

Following biofilm formation in 96-well microtiter plates and quantification by crystal violet staining, a significant difference was observed between $P$. aeruginosa PAO1 control biofilms and biofilms grown in the presence of MomL (concentration $>50 \mu \mathrm{g} / \mathrm{mL}$ ) (Fig. 3A). When grown with $150 \mu \mathrm{g} / \mathrm{mL}$ MomL, an average decrease of approximately $35 \%$ was observed. MomL inhibited A. baumannii LMG 10531 biofilm formation at concentrations as low as $0.1 \mu \mathrm{g} / \mathrm{mL}$, and the biofilm biomass was reduced by approximately $42 \%$ when exposed to $5 \mu \mathrm{g} / \mathrm{mL}$ MomL (Fig. 3B). No further decrease was observed when A. baumannii LMG 10531 biofilms were grown in the presence of higher concentration of MomL.

\section{Effect of MomL on biofilm susceptibility to antibiotics}

Application of MomL alone $(200 \mu \mathrm{g} / \mathrm{mL}$ for $P$. aeruginosa PAO1 and $10 \mu \mathrm{g} / \mathrm{mL}$ for $A$. baumannii LMG 10531) reduced the number of cultivable biofilm cells by approximately $50 \%$ in both $P$. aeruginosa $\mathrm{PAO} 1$ and $A$. baumannii LMG 10531. For $P$. aeruginosa PAO1, combining CIP or MEM with MomL led to $>70 \%$ more reduction compared to treatment with CIP or MEM alone (Fig. 4A). For A. baumannii LMG 10531, MomL also increased killing of biofilm cells when antibiotics were used together with MomL (Fig. 4B). In case of TOB, cell number was reduced by $80 \%$ when used in combination with MomL compared to TOB alone. Consistent with results obtained by plating, fewer living cells were observed in fluorescence microscope images of biofilms treated with MomL, TOB, or a combination of both, compared to control biofilms (Fig. 5). 
236

237

238

239

240

241

242

243

244

245

246

247

248

249

250

251

252

253

254

255

256

257

258

259

260

261

262

263

\section{Effect of MomL on dual-species biofilm formed by $P$. aeruginosa and $A$. baumannii}

We also evaluated the effect of MomL on dual-species biofilm formed by P. aeruginosa PAO1 and $A$. baumannii LMG 10531. We found that $P$. aeruginosa PAO1 inhibited growth of $A$. baumannii LMG 10531 in dual-species biofilm, and most $A$. baumannii LMG 10531 cells were killed by P. aeruginosa PAO1 after 48h (Fig. 6). When MomL was added, there was a reduction in A. baumannii LMG 10531 cell numbers; however no difference was observed in either total cell numbers or number of surviving $P$. aeruginosa PAO1 cells (Fig. 6A). MomL in combination of TOB was also tested, but no change in susceptibility to TOB was observed in the dual-species biofilm (Fig. 6B).

\section{Effect of MomL on other Acinetobacter strains}

We also tested MomL on four other Acinetobacter strains. However, only A. baumannii LMG 10520 showed reduction in biofilm biomass when treated with MomL at $50 \mu \mathrm{g} / \mathrm{mL}$ (Fig. 7). No significant difference was observed for A. calcoaceticus LMG 10517, A. nosocomialis M2 and A. baumannii AB5075. The effect of MomL on susceptibility of A. baumannii LMG 10520 and $A$. calcoaceticus LMG 10517 biofilms was also tested. For A. baumannii LMG 10520, significant differences were detected when MomL was added alone or in combination with several antibiotics (Fig. 8). For A. calcoaceticus LMG 10517, no difference was observed between biofilms receiving MomL treatment and biofilms receiving the control treatment, either by plating or fluorescence microscope.

\section{Effect of MomL in a biofilm wound model system and in the $C$. elegans model}

An in vitro wound model was used to mimic the conditions in an infected wound. For both $P$. aeruginosa PAO1 and A. baumannii LMG 10531, MomL had no effect on biofilm formation in this wound model (Fig. 9).

The $C$. elegans model was used to further evaluate whether MomL can increase survival of nematodes infected with $A$. baumannii. However, no significant increase of $C$. elegans survival was found after treating nematodes infected with A. baumannii LMG 10520 or A. baumannii 
264 LMG 10531 with MomL (Fig. 10), although AHL-degrading activity was maintained under these 265 test conditions (Fig. S2).

\section{Discussion}

267 QS disruption has been considered as a promising anti-infectious strategy to substitute or at least 268 supplement treatment with antibiotics, and could inhibit production of virulence factors and the 269 formation of biofilms (Brackman et al., 2011). Compared to QS inhibitors, QQ enzymes can 270 degrade AHLs from different pathogens and might be more effective in treating multispecies

271 infections. In addition, QQ enzymes do not need to enter the cells as they can act extracellularly, 272 making it less likely resistance will develop (Bzdrenga et al. 2016). The recently-discovered QQ 273 enzyme, MomL, has strong degrading activity towards AHLs with different acyl-chain length 274 and substituents (oxo or hydroxyl) (Tang et al. 2015), and this could be an advantage when 275 targeting bacteria like Acinetobacter strains that produce various AHLs. MomL was reported to 276 reduce the in vitro production of protease and pyocyanin by $P$. aeruginosa and attenuate the 277 virulence of $P$. aeruginosa in a C. elegans infection model (Tang et al. 2015). The further 278 application potential of MomL was not determined yet. In the present study we investigated the 279 possible use of MomL for treating biofilm infections, and evaluate its effect on two important 280 Gram-negative nosocomial pathogens, P. aeruginosa and A. baumannii in different models.

281 First we tested the effect of MomL on single-species biofilms of $P$. aeruginosa PAO1 and $A$.

282 baumannii LMG 10531 formed in microtiter plates; a reduction of biofilm biomass was observed 283 for both strains. The maximum decrease in biofilm of $A$. baumannii LMG 10531 was achieved at 284 a concentration of $5 \mu \mathrm{g} / \mathrm{mL}$ and no further decrease was observed with higher concentrations of 285 MomL, which indicated the presence of other mechanisms besides QS regulating biofilm 286 formation in A. baumannii. Higher concentrations of MomL are needed to show an effect on 287 biofilm of $P$. aeruginosa PAO1 comparing to A. baumannii LMG 10531 (Figure 2), possibly due 288 to the higher concentration of AHLs produced by PAO1. When used in combination with 289 antibiotics, fewer biofilm cells survived compared to antibiotic treatment alone, both for $P$. aeruginosa PAO1 and A. baumannii LMG 10531. In addition, MomL showed no inhibition on 291 planktonic cells of both P. aeruginosa and A. baumannii (Fig. S1), and all these in vitro results 292 seem promising and suggest possible use of MomL to treat biofilm infections of $P$. aeruginosa 293 and A. baumannii. 
294 We subsequently investigated the effect of MomL in a dual-species biofilm formed by $P$.

295 aeruginosa and $A$. baumannii and in a wound biofilm model. MomL had no effect on the overall 296 cell number in the mixed species biofilm and the same disappointing results were obtained in

297 biofilms formed in wound model system. In this wound model system, medium containing

298 plasma, serum, horse blood and heparin was used to reflect nutritional condition in wounds. An 299 artificial dermis was used to mimic a wound like surface and an inoculum of $10^{4}$ cells was used 300 to reflect the microbial load of a wound prior to infection. Additionally, in contrast to what we 301 observed for the mono-species biofilms formed in 96-well microtiter plates, MomL did not 302 potentiate the activity of TOB in this model system. Possible explanations for this are that 303 component(s) present in this wound biofilm model protect AHL from degradation and/or interfere with the activity of MomL (potentially through interactions with proteins in the plasma), or that QS is not essential for biofilm formation and/or resistance in these conditions. Further experiments will be required to clarify this. Although MomL showed strong activity against 3$\mathrm{OH}-\mathrm{C} 12-\mathrm{HSL}$ in the medium used in the C. elegans model (Fig. S2), no effect of MomL on the virulence of $A$. baumanii was observed. As previously reported, an A. baumannii QS mutant did not differ from the wild type with regards to killing in a Galleria mellonella infection model (Peleg et al. 2009). These results indicated that although QS is known to play an important role in $A$. baumanii biofilm formation, it might only have limited role in the virulence in the $C$. elegans and G. mellonella models.

Thus far, a series of promising results about in vivo application of QQ enzymes have been reported. Phosphotriesterase-like lactonase SsoPox-I has been reported to reduce biofilm formation of $P$. aeruginosa at a concentration higher than $170 \mu \mathrm{g} / \mathrm{mL}$, and the early use of SsoPox-I reduced the mortality of rats with acute pneumonia from $75 \%$ to $20 \%$ (Hraiech et al. 2014). In another study, acylase-containing coatings on silicone urinary catheters reduced formation of $P$. aeruginosa biofilms and mixed-species $P$. aeruginosa $-E$. coli biofilms (Ivanova et al. 2015). Our data obtained in a dual-species biofilm formed by $P$. aeruginosa and $A$. baumannii as well as in a wound model strongly suggest that the effect of MomL (and potentially also other QQ enzymes) on in vivo grown bacterial biofilms may be much less pronounced than the effect observed with biofilms formed under simple in vitro conditions. 
324 enzyme, penetration of the enzyme through the biofilm matrix, and the composition of the 325 environment.

326 Different outcomes were also observed when we evaluated the effect of MomL on different 327 Acinetobacter strains, and no effects of MomL on biofilm formation was detected for three out of 328 five Acinetobacter strains tested. In addition, for $A$. baumanii LMG 10520, a considerably higher 329 concentration of MomL was required to obtain a pronounced inhibitory effect than for $A$. 330 baumannii LMG 10531. These results confirm that the anti-biofilm activity of QQ enzymes is 331 strain-dependent, which is likely to reduce their clinical efficacy.

\section{Conclusion}

334 335

The results of the present study highlight that there are considerable hurdles to be cleared before QQ enzymes could potentially be used to combat infections. Our data indicate that demonstrating AHL degrading activity in vitro and/or anti-biofilm activity in simple in vitro biofilm model systems is not sufficient to predict an anti-biofilm effect in more complex systems.

\section{Acknowledgements}

We thank prof. Xiao-Hua Zhang for providing Escherichia coli BL21(DE3) harboring the MomL expression plasmid pET24a(+)-momL-(-SP), prof. Wim Quax for providing $A$. nosocomialis M2 and prof. Colin Manoil for providing A. baumannii AB5075.

\section{References}

Aybey A, and Demirkan E. 2016. Inhibition of quorum sensing-controlled virulence factors in Pseudomonas aeruginosa by human serum paraoxonase. Journal of Medical Microbiology 65:105-113.

Bassler BL, and Losick R. 2006. Bacterially speaking. Cell 125:237-246. 
349

350

351

352

353

354

355

356

357

358

359

360

361

362

363

364

365

366

367

368

369

370

371

372

373

374

375

376

377

Bhargava N, Sharma P, and Capalash N. 2010. Quorum sensing in Acinetobacter: an emerging pathogen. Critical Reviews in Microbiology 36:349-360.

Bhargava N, Singh SP, Sharma A, Sharma P, and Capalash N. 2015. Attenuation of quorum sensing-mediated virulence of Acinetobacter baumannii by Glycyrrhiza glabra flavonoids. Future Microbiology 10:1953-1968.

Brackman G, and Coenye T. 2015. Quorum sensing inhibitors as anti-biofilm agents. Current Pharmaceutical Design 21:5-11.

Brackman G, Cos P, Maes L, Nelis HJ, and Coenye T. 2011. Quorum sensing inhibitors increase the susceptibility of bacterial biofilms to antibiotics in vitro and in vivo. Antimicrob Agents Chemother 55:2655-2661. 10.1128/AAC.00045-11

Brackman G, Garcia-Fernandez MJ, Lenoir J, De Meyer L, Remon JP, De Beer T, Concheiro A, Alvarez-Lorenzo C, and Coenye T. 2016. Dressings loaded with cyclodextrinhamamelitannin complexes increase Staphylococcus aureus susceptibility toward antibiotics both in single as well as in mixed biofilm communities. Macromolecular Bioscience.

Bzdrenga J, Daudé D, Rémy B, Jacquet P, Plener L, Elias M, and Chabrière E. 2016. Biotechnological applications of quorum quenching enzymes. Chemico-Biological Interactions.

Chow JY, Yang Y, Tay SB, Chua KL, and Yew WS. 2014. Disruption of biofilm formation by the human pathogen Acinetobacter baumannii using engineered quorum-quenching lactonases. Antimicrob Agents Chemother 58:1802-1805.

Donlan RM, and Costerton JW. 2002. Biofilms: survival mechanisms of clinically relevant microorganisms. Clinical Microbiology Reviews 15:167-193. 10.1128/cmr.15.2.167-193.2002

Fetzner S. 2015. Quorum quenching enzymes. Journal of Biotechnology 201:2-14.

Furiga A, Lajoie B, El Hage S, Baziard G, and Roques C. 2016. Impairment of Pseudomonas aeruginosa biofilm resistance to antibiotics by bombining the drugs with a new quorumsensing inhibitor. Antimicrob Agents Chemother 60:1676-1686.

Gonzalez-Villoria AM, and Valverde-Garduno V. 2016. Antibiotic-resistant Acinetobacter baumannii increasing success remains a challenge as a nosocomial pathogen. Journal of pathogens 2016. 
378 González R, Dijkshoorn L, Van den Barselaar M, and Nudel C. 2009. Quorum sensing signal 379 profile of Acinetobacter strains from nosocomial and environmental sources. Rev Argent $380 \quad$ Microbiol 41:73-78.

381

Hall-Stoodley L, Costerton JW, and Stoodley P. 2004. Bacterial biofilms: from the natural environment to infectious diseases. Nat Rev Microbiol 2:95-108. 10.1038/nrmicro821

Hentzer M, and Givskov M. 2003. Pharmacological inhibition of quorum sensing for the treatment of chronic bacterial infections. The Journal of clinical investigation 112:1300-1307. Hentzer M, Riedel K, Rasmussen TB, Heydorn A, Andersen JB, Parsek MR, Rice SA, Eberl L, Molin S, and Høiby N. 2002. Inhibition of quorum sensing in Pseudomonas aeruginosa biofilm bacteria by a halogenated furanone compound. Microbiology 148:87-102.

Hentzer M, Wu H, Andersen JB, Riedel K, Rasmussen TB, Bagge N, Kumar N, Schembri MA, Song Z, and Kristoffersen P. 2003. Attenuation of Pseudomonas aeruginosa virulence by quorum sensing inhibitors. The EMBO journal 22:3803-3815.

Hraiech S, Hiblot J, Lafleur J, Lepidi H, Papazian L, Rolain J-M, Raoult D, Elias M, Silby MW, and Bzdrenga J. 2014. Inhaled lactonase reduces Pseudomonas aeruginosa quorum sensing and mortality in rat pneumonia. PLoS One 9:e107125.

Ivanova K, Fernandes MM, Francesko A, Mendoza E, Guezguez J, Burnet M, and Tzanov T. 2015. Quorum-quenching and matrix-degrading enzymes in multilayer coatings synergistically prevent bacterial biofilm formation on urinary catheters. ACS applied materials \& interfaces 7:27066-27077.

LaSarre B, and Federle MJ. 2013. Exploiting quorum sensing to confuse bacterial pathogens. Microbiology and Molecular Biology Reviews 77:73-111.

Longo F, Vuotto C, and Donelli G. 2014. Biofilm formation in Acinetobacter baumannii. New Microbiologica 37:119-127.

Migiyama Y, Kaneko Y, Yanagihara K, Morohoshi T, Morinaga Y, Nakamura S, Miyazaki T, Hasegawa H, Izumikawa K, and Kakeya H. 2013. Efficacy of AiiM, an N-acylhomoserine lactonase, against Pseudomonas aeruginosa in a mouse model of acute pneumonia. Antimicrob Agents Chemother 57:3653-3658.

Niu C, Clemmer KM, Bonomo RA, and Rather PN. 2008. Isolation and characterization of an autoinducer synthase from Acinetobacter baumannii. Journal of Bacteriology 190:3386-3392. 
408 O'Loughlin CT, Miller LC, Siryaporn A, Drescher K, Semmelhack MF, and Bassler BL. 2013.

409 A quorum-sensing inhibitor blocks Pseudomonas aeruginosa virulence and biofilm formation.

$410 \quad$ Proceedings of the National Academy of Sciences 110:17981-17986.

411 Peeters E, Nelis HJ, and Coenye T. 2008. Comparison of multiple methods for quantification of

412 microbial biofilms grown in microtiter plates. Journal of Microbiological Methods 72:157$413 \quad 165$.

414 Peleg AY, Jara S, Monga D, Eliopoulos GM, Moellering RC, and Mylonakis E. 2009. Galleria

415 mellonella as a model system to study Acinetobacter baumannii pathogenesis and therapeutics.

416 Antimicrob Agents Chemother 53:2605-2609.

417 Peleg AY, Seifert H, and Paterson DL. 2008. Acinetobacter baumannii: emergence of a 418 successful pathogen. Clinical Microbiology Reviews 21:538-582.

419 Pesci EC, Pearson JP, Seed PC, and Iglewski BH. 1997. Regulation of las and rhl quorum 420 sensing in Pseudomonas aeruginosa. Journal of Bacteriology 179:3127-3132.

421 Potron A, Poirel L, and Nordmann P. 2015. Emerging broad-spectrum resistance in

422 Pseudomonas aeruginosa and Acinetobacter baumannii: mechanisms and epidemiology.

423 International Journal of Antimicrobial Agents 45:568-585.

424 Rasmussen TB, and Givskov M. 2006. Quorum sensing inhibitors: a bargain of effects.

425 Microbiology 152:895-904.

426 Rutherford ST, and Bassler BL. 2012. Bacterial quorum sensing: its role in virulence and

427 possibilities for its control. Cold Spring Harbor Perspectives in Medicine 2:a012427.

428 Saroj SD, and Rather PN. 2013. Streptomycin inhibits quorum sensing in Acinetobacter

429 baumannii. Antimicrob Agents Chemother 57:1926-1929.

430 Stiernagle T. 1999. Maintenance of C. elegans. C elegans 2:51-67.

431 Tang K, Su Y, Brackman G, Cui F, Zhang Y, Shi X, Coenye T, and Zhang X-H. 2015. MomL, a

432 novel marine-derived N-acyl homoserine lactonase from Muricauda olearia. Applied and

433 Environmental Microbiology 81:774-782.

434 Tang K, and Zhang X-H. 2014. Quorum quenching agents: resources for antivirulence therapy.

435 Marine Drugs 12:3245-3282.

436 Tang K, Zhang Y, Yu M, Shi X, Coenye T, Bossier P, and Zhang X-H. 2013. Evaluation of a

437 new high-throughput method for identifying quorum quenching bacteria. Scientific reports

$438 \quad 3: 2935$. 
439 Tempé J, Petit A, Holsters M, Van Montagu M, and Schell J. 1977. Thermosensitive step

440 associated with transfer of the Ti plasmid during conjugation: possible relation to

441 transformation in crown gall. Proceedings of the National Academy of Sciences 74:2848-2849.

443 Williams P, Winzer K, Chan WC, and Camara M. 2007. Look who's talking: communication and 444 quorum sensing in the bacterial world. Philosophical Transactions of the Royal Society B:

445 Biological Sciences 362:1119-1134.

446 Wu H, Song Z, Hentzer M, Andersen JB, Molin S, Givskov M, and Høiby N. 2004. Synthetic

447 furanones inhibit quorum-sensing and enhance bacterial clearance in Pseudomonas

448 aeruginosa lung infection in mice. Journal of Antimicrobial Chemotherapy 53:1054-1061.

449 Yin H, Deng Y, Wang H, Liu W, Zhuang X, and Chu W. 2015. Tea polyphenols as an

450 antivirulence compound disrupt quorum-sensing regulated pathogenicity of Pseudomonas

451 aeruginosa. Scientific reports 5.

452 Zhu J, Beaber JW, Moré MI, Fuqua C, Eberhard A, and Winans SC. 1998. Analogs of the

453 autoinducer 3-oxooctanoyl-homoserine lactone strongly inhibit activity of the TraR protein of

454 Agrobacterium tumefaciens. Journal of bacteriology 180:5398-5405. 
Figure 1

SDS-PAGE of purified MomL.

Lane1, molecular mass markers; Lane 2, purified recombinant MomL with molecular mass of nearly $31 \mathrm{kDa}$. 
200

116

97

66

55

45

36

29

- MomL

24 -

$20-$ 
Figure 2

Degradation of 3-OH- $\mathrm{C}_{12}-\mathrm{HSL}$ by MomL.

The amount of residual 3-OH-C12-HSL was expressed as the normalized $\beta$-galactosidase activity. Data shown are average of three technical replicates $(n=3)$, error bars represent standard deviation. *, $\mathrm{P}<0.05$ when compared with non-MomL treated control (Kruskal-Wallis test).

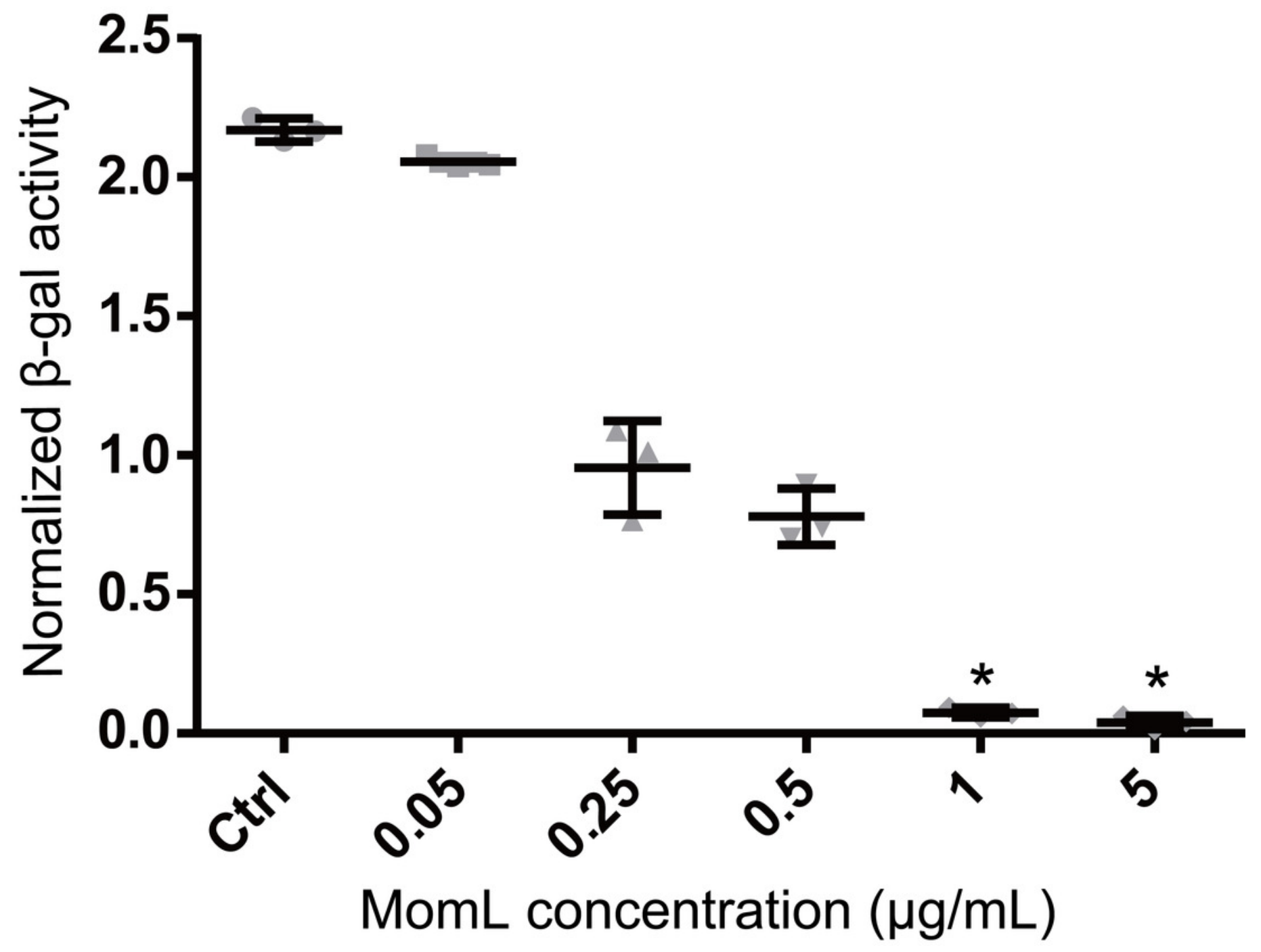


Figure 3

Effect of MomL on biofilm formation by P. aeruginosa PAO1 (A) and A. baumannii LMG 10531 (B) .

Biofilms were quantified by CV staining and amount of biofilm left is expressed as CV absorbance (OD 590). Data shown are average of three biological replicates with variable numbers of technical replicates each ( $n \geq 27$ ), error bars represent standard deviation. *, $\mathrm{P}<0.05$ when compared with non-MomL treated control in Kruskal-Wallis test (A) or one-way ANOVA(B).

A.

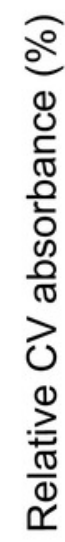

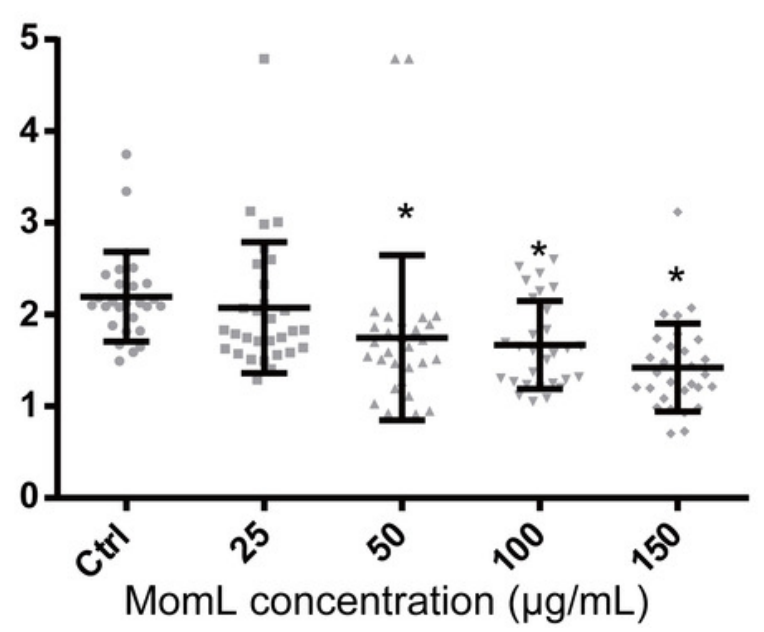

B.

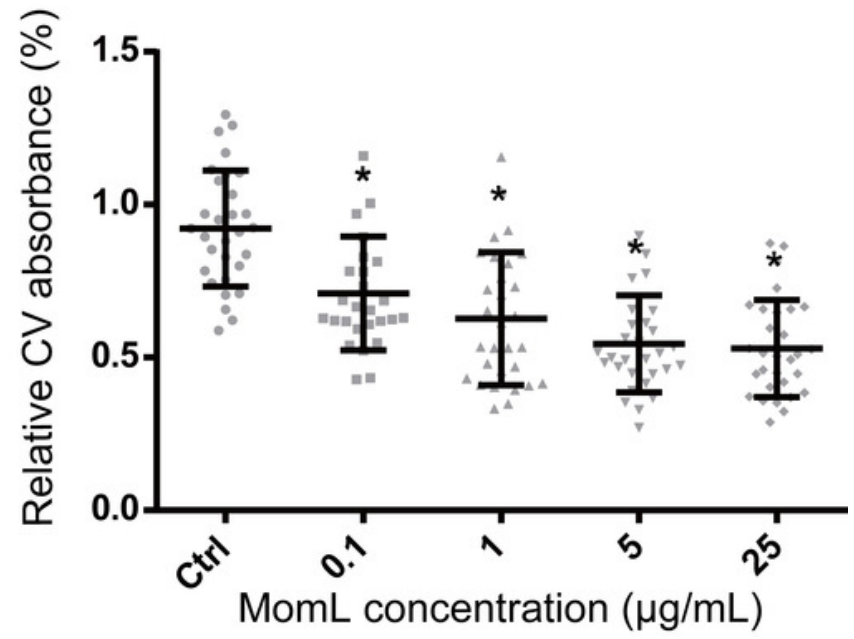


Figure 4

Effect of MomL on susceptibility of $P$. aeruginosa PAO1 (A) and A. baumannii LMG 10531 (B) biofilms to different antibiotics.

Numbers of CFU/biofilm were determined by plating and shown as box-whisker plots. Boxes span the interquartile range; the line within each box denotes the median, and whiskers indicate the minimum and maximum values. MomL was added in a final concentration of 200 $\mu \mathrm{g} / \mathrm{mL}$ for $P$. aeruginosa PAO1 and $10 \mu \mathrm{g} / \mathrm{mL}$ for $A$. baumannii LMG 10531. Data shown are from three biological replicates with three technical replicates each $(n=9)$. Mann-Whitney tests were performed to compare different groups $(*, \mathrm{P}<0.05)$

A.

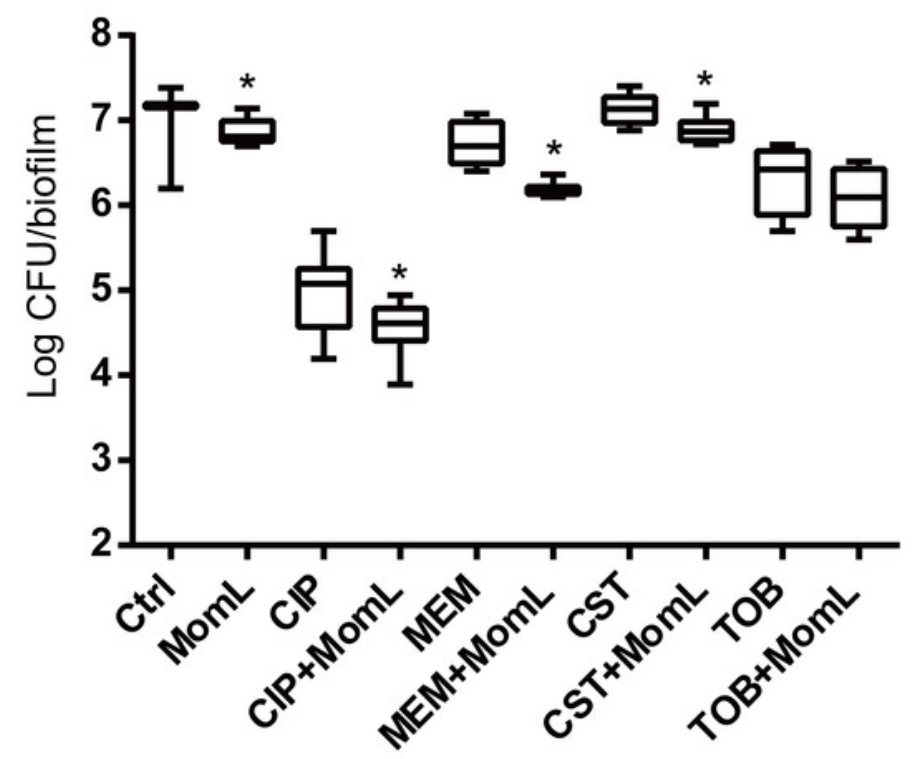

B.

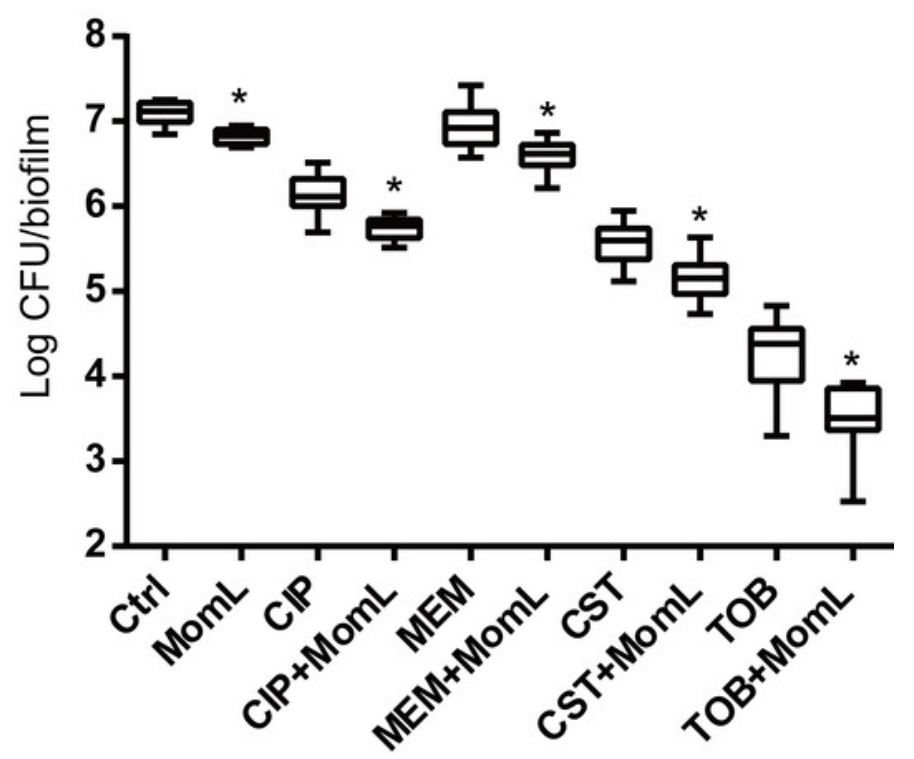




\section{Figure 5}

Representative fluorescence images of biofilms of $P$. aeruginosa PAO1 and $A$. baumannii LMG 10531.

Biofilms were treated with MomL alone, TOB alone or a combination of both and stained with Syto9 and propidium iodide. $40 \times$ Objective (numerical aperture: 0.75 ) was used and the final magnification is $1200 \times$. The scale bar represents $100 \mu \mathrm{m}$.

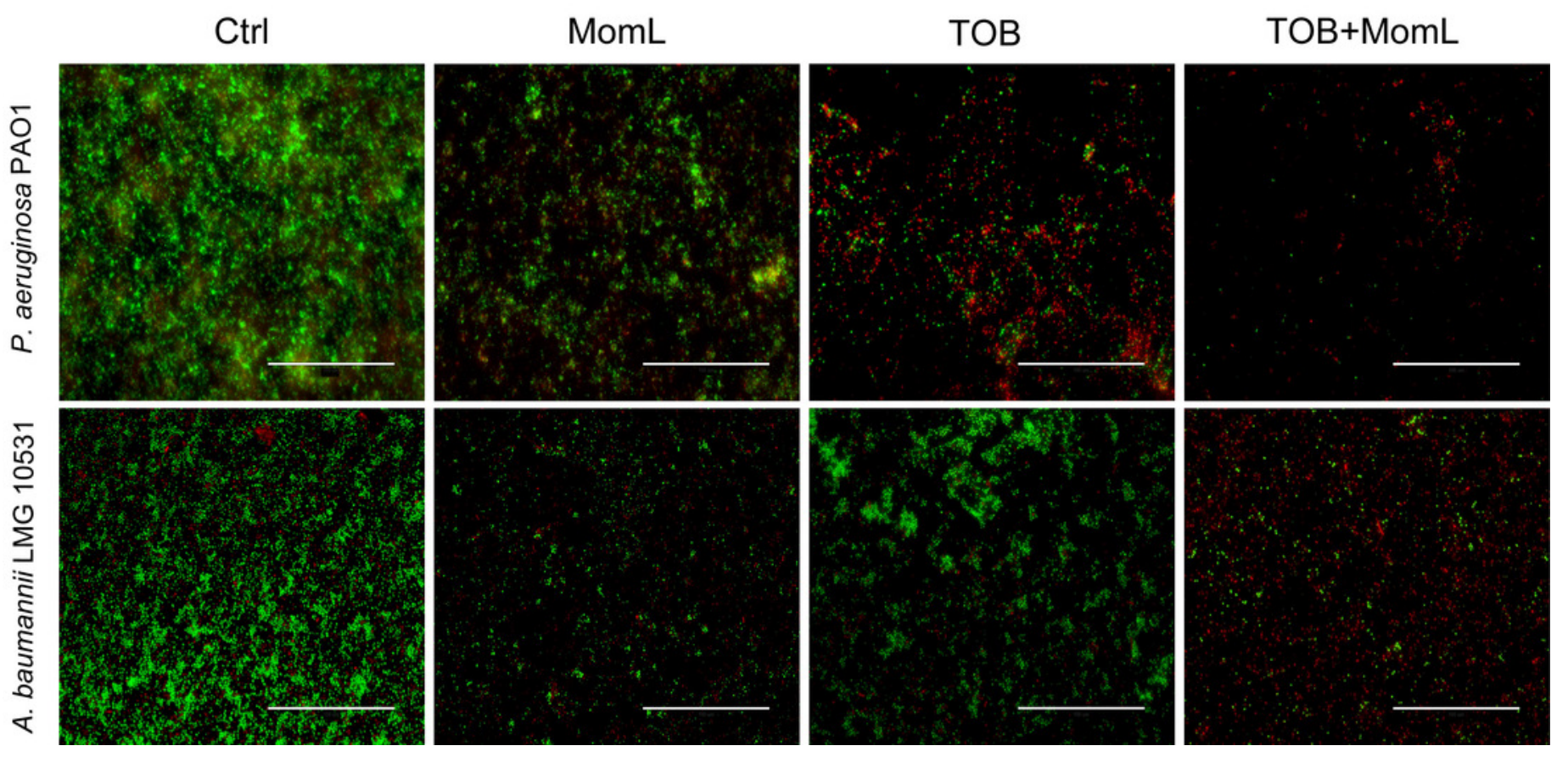




\section{Figure 6}

Effect of MomL on dual-species biofilms.

Total number of CFU/biofilm, number of $P$. aeruginosa PAO1 CFU/biofilm and number of $A$. baumannii LMG 10531 CFU/biofilm in each dual-species biofilm were determined by plating and shown as box-whisker plots. Boxes span the interquartile range; the line within each box denotes the median, and whiskers indicate the minimum and maximum values. (A). 24hbiofilm treated with MomL alone; (B). 48h-biofilm treated with MomL alone, TOB alone or a combination of both. Data shown are from three biological replicates with three $(A)$ or two (B) technical replicates each ( $n=9$ for $A, n=6$ for $B$ ). Mann-Whitney tests were performed to compare total, $P$. aeruginosa PAO1 and A. baumannii LMG 10531 cell numbers respectively between untreated or MomL-treated dual-species biofilm $(*, P<0.05)$.

A.

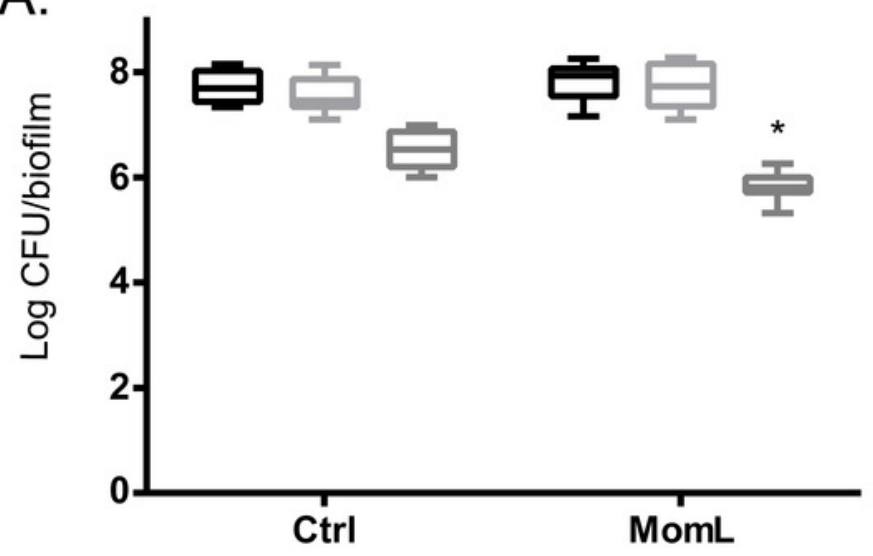

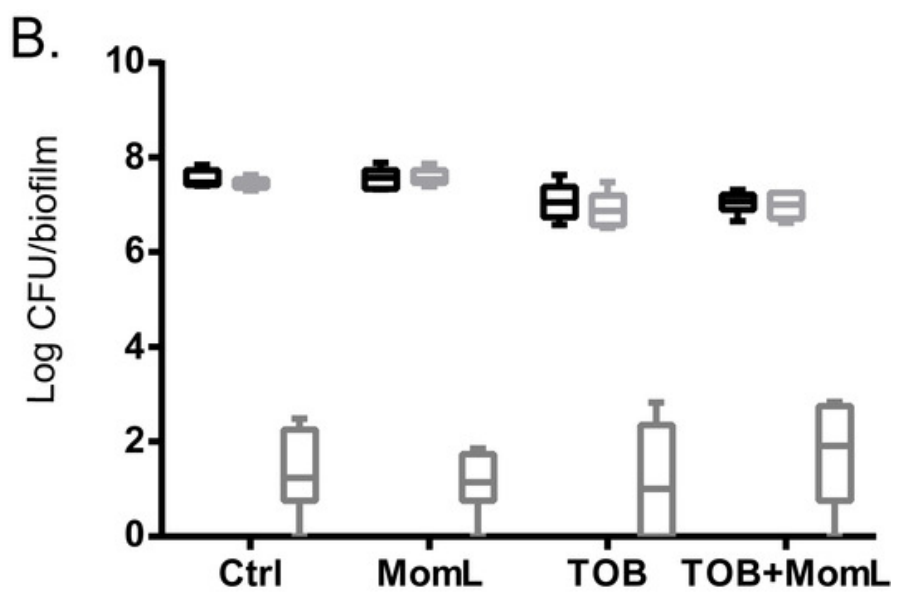

A.baumannii LMG 10531 


\section{Figure 7}

Effect of MomL on biofilms formed by other Acinetobacter strains.

Biofilms of $A$. calcoaceticus LMG 10517, A. nosocomialis M2, A. baumannii LMG 10520 and $A$. baumannii AB5075 were treated with different concentration of MomL and quantified by $\mathrm{CV}$ staining. Data shown in box-whisker plots are from three biological replicates with variable numbers of technical replicates each ( $n \geq 27$ ). Boxes span the interquartile range; the line within each box denotes the median, and whiskers indicate the minimum and maximum values. *, P<0.05 when compared to untreated control (Kruskal-Wallis test).

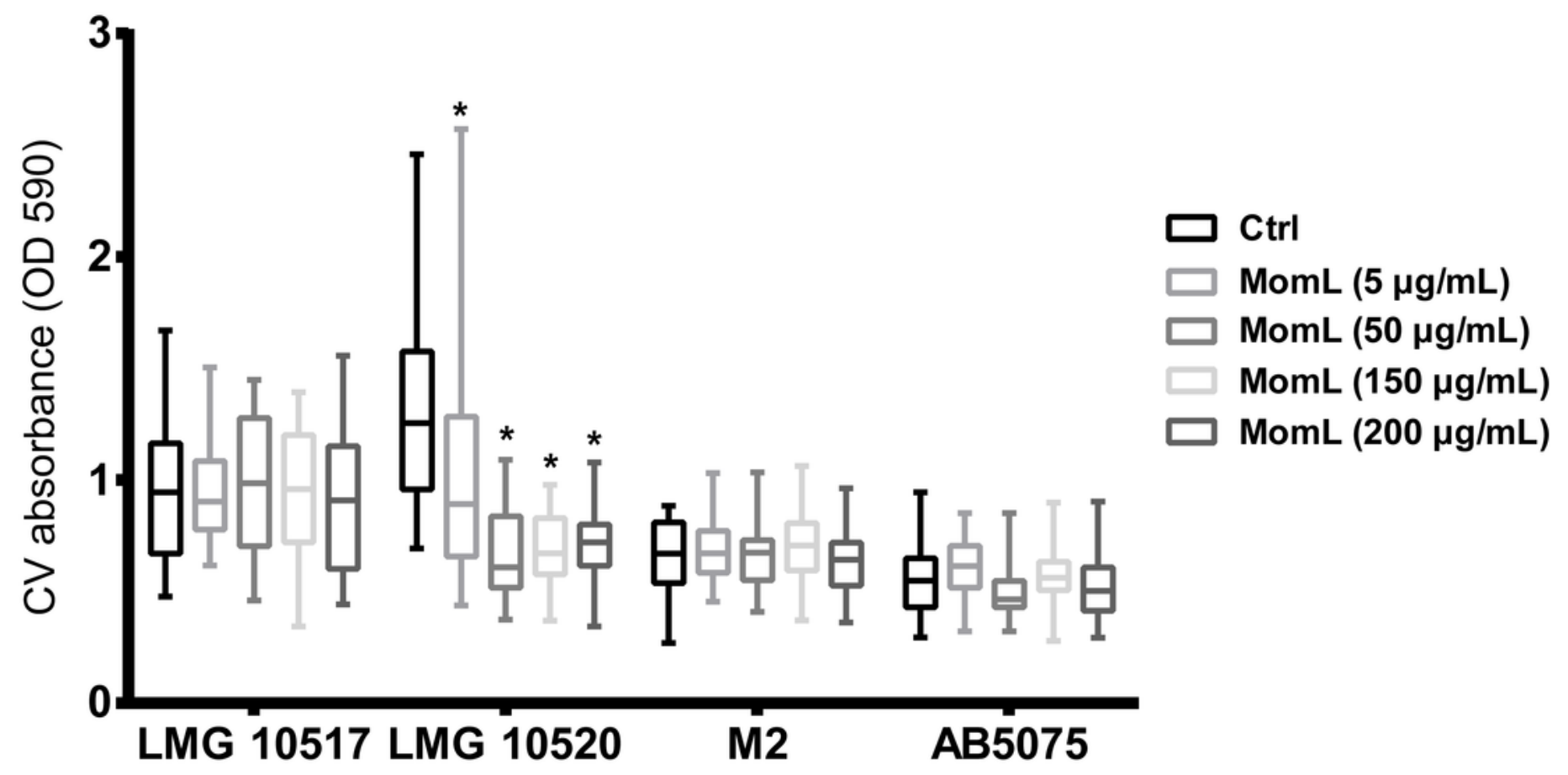




\section{Figure 8}

Effect of MomL on biofilm susceptibility of $A$. baumannii LMG 10520 and A. calcoaceticus LMG 10517.

(A). Plating results for biofilms of $A$. baumannii LMG 10520 exposed to CIP, MEM, CST, TOB alone or in combination with MomL (50 $\mu \mathrm{g} / \mathrm{mL})$; (B), Plating results for biofilms of $A$. calcoaceticus LMG 10517 exposed to CIP, MEM, CST, TOB alone or in combination with MomL $(200 \mu \mathrm{g} / \mathrm{mL}$ ). Data shown in box-whisker plots are from two biological replicates with three technical replicates each $(n=6)$. Boxes span the interquartile range; the line within each box denotes the median, and whiskers indicate the minimum and maximum values. MannWhitney tests were performed to compare control and MomL or antibiotic treatment alone and in combination with MomL $(*, \mathrm{P}<0.05)$. (C). Representative fluorescence images of $A$. baumannii LMG 10520 and A. calcoaceticus LMG 10517. Biofilms were treated with MomL alone or in combination with tobramycin and stained with Syto9 and propidium iodide. $20 \mathrm{x}$ Objective (numerical aperture: 0.65 ) was used and the final magnification is 599x. The scale bar represents $200 \mu \mathrm{m}$. 

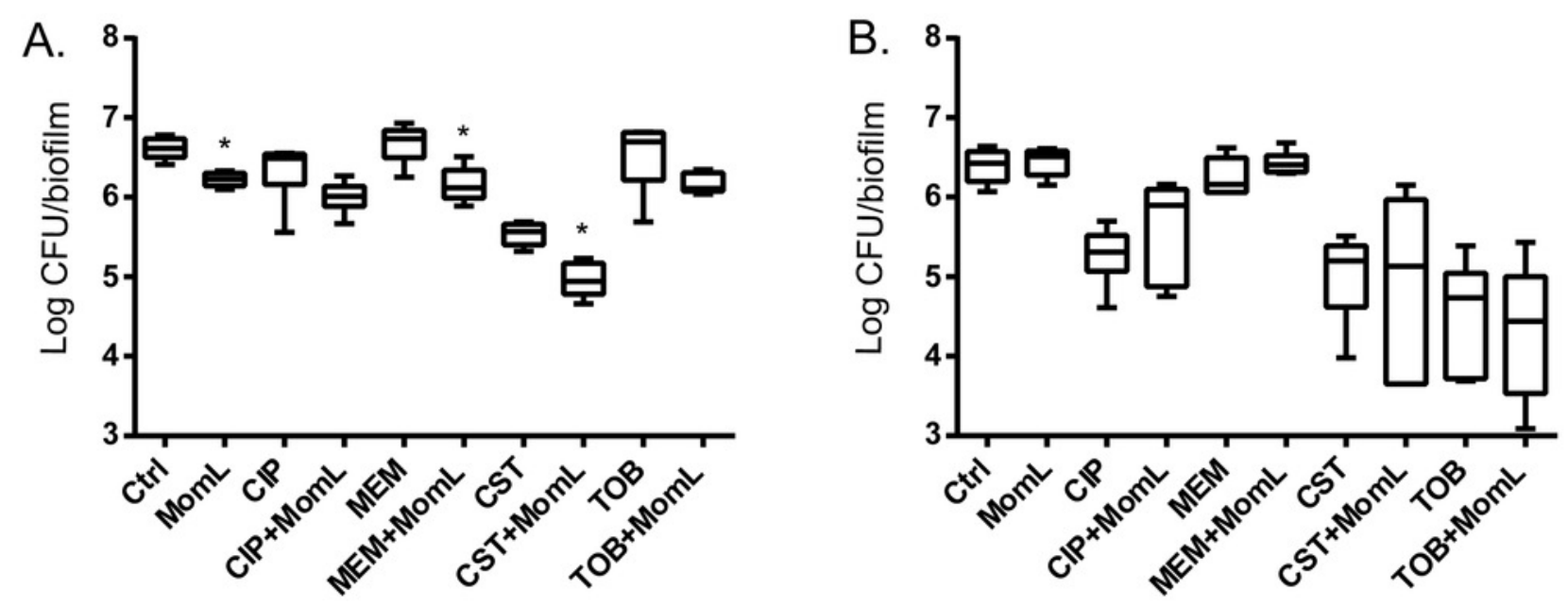

C.

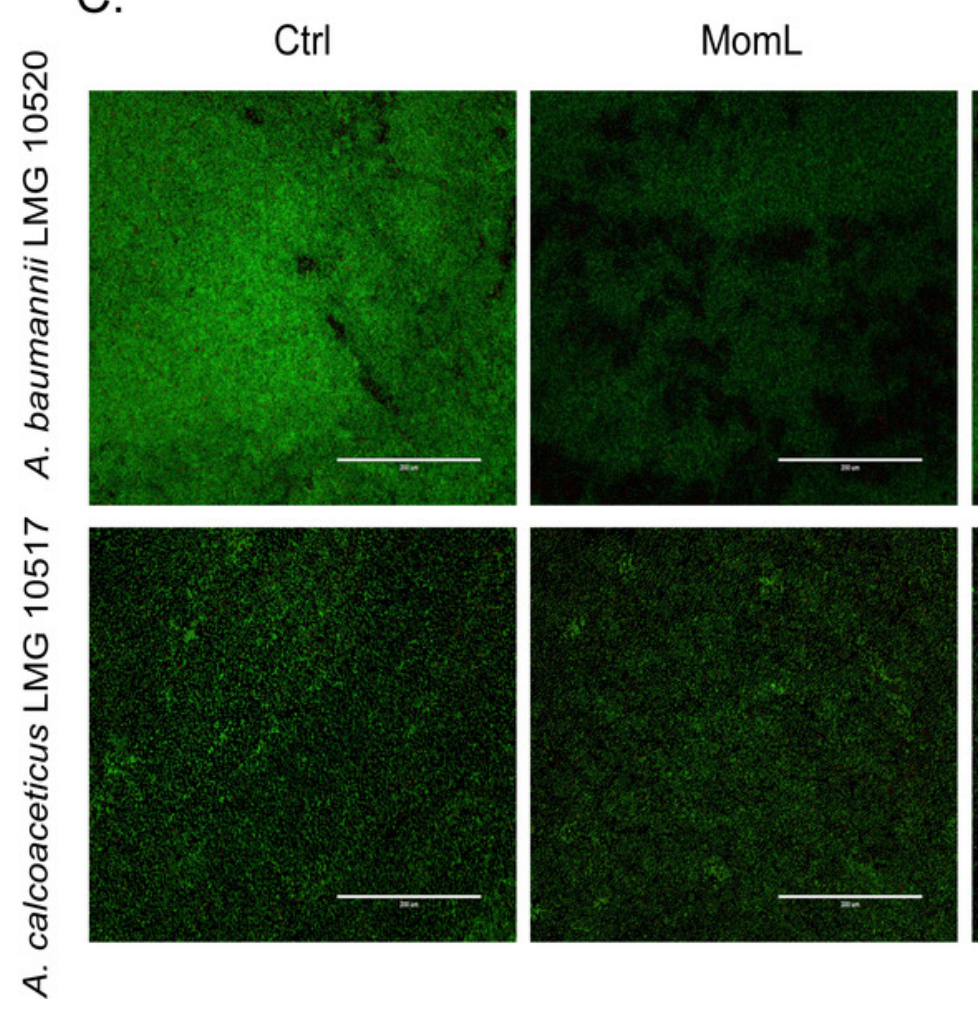

TOB
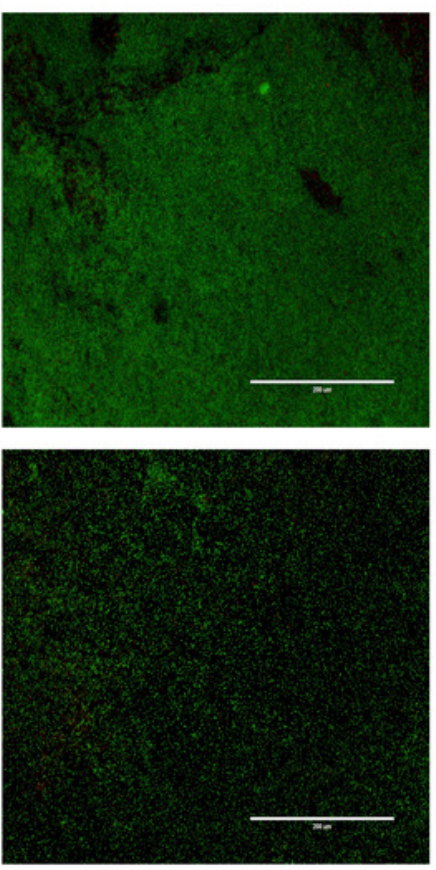

TOB+MomL
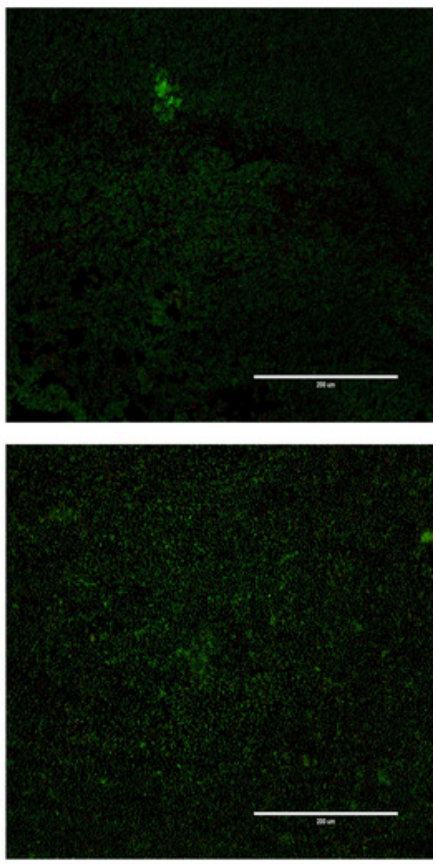
Figure 9

Effect of MomL on biofilms of $P$. aeruginosa PAO1 and A. baumannii LMG 10531 formed in wound model.

Data shown in box-whisker plots are from three biological replicates with two technical replicates each $(n=6)$. Boxes span the interquartile range; the line within each box denotes the median, and whiskers indicate the minimum and maximum values. Mann-Whitney tests were performed to compare control and MomL treatment, or TOB and TOB in combination with MomL.

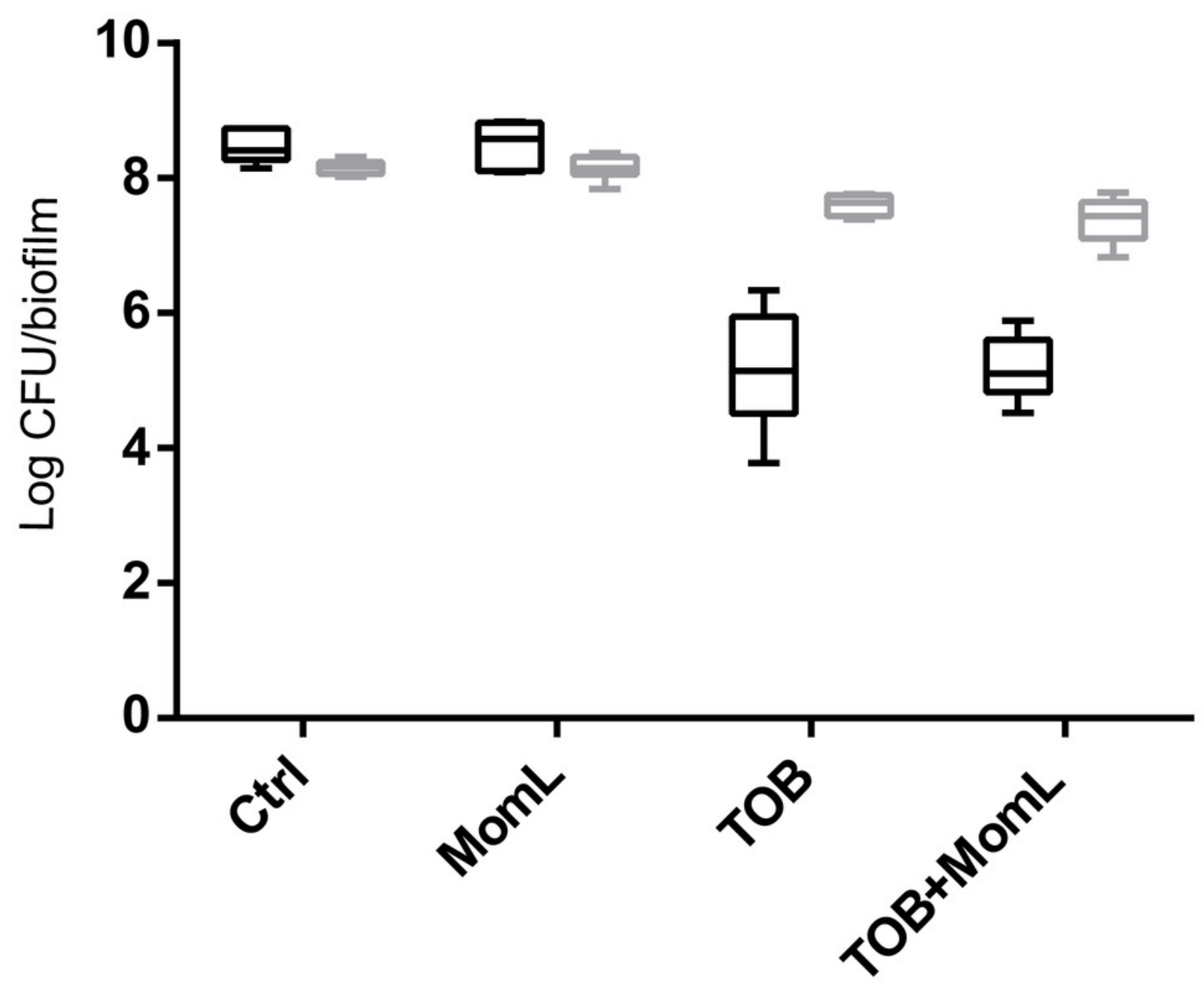




\section{Figure 10}

Effect of MomL on the virulence of $A$. baumanii strains in $C$. elegans.

Percent survival of $C$. elegans infected by $A$. baumannii LMG 10520 and LMG 10531. Data shown in box-whisker plots are from three biological replicates with three technical replicates each $(n=9)$. Boxes span the interquartile range; the line within each box denotes the median, and whiskers indicate the minimum and maximum values. One-way ANOVA was performed, and no significant differences were found between control and MomL treatment in both uninfected $C$. elegans and those infected by $A$. baumannii strains. 


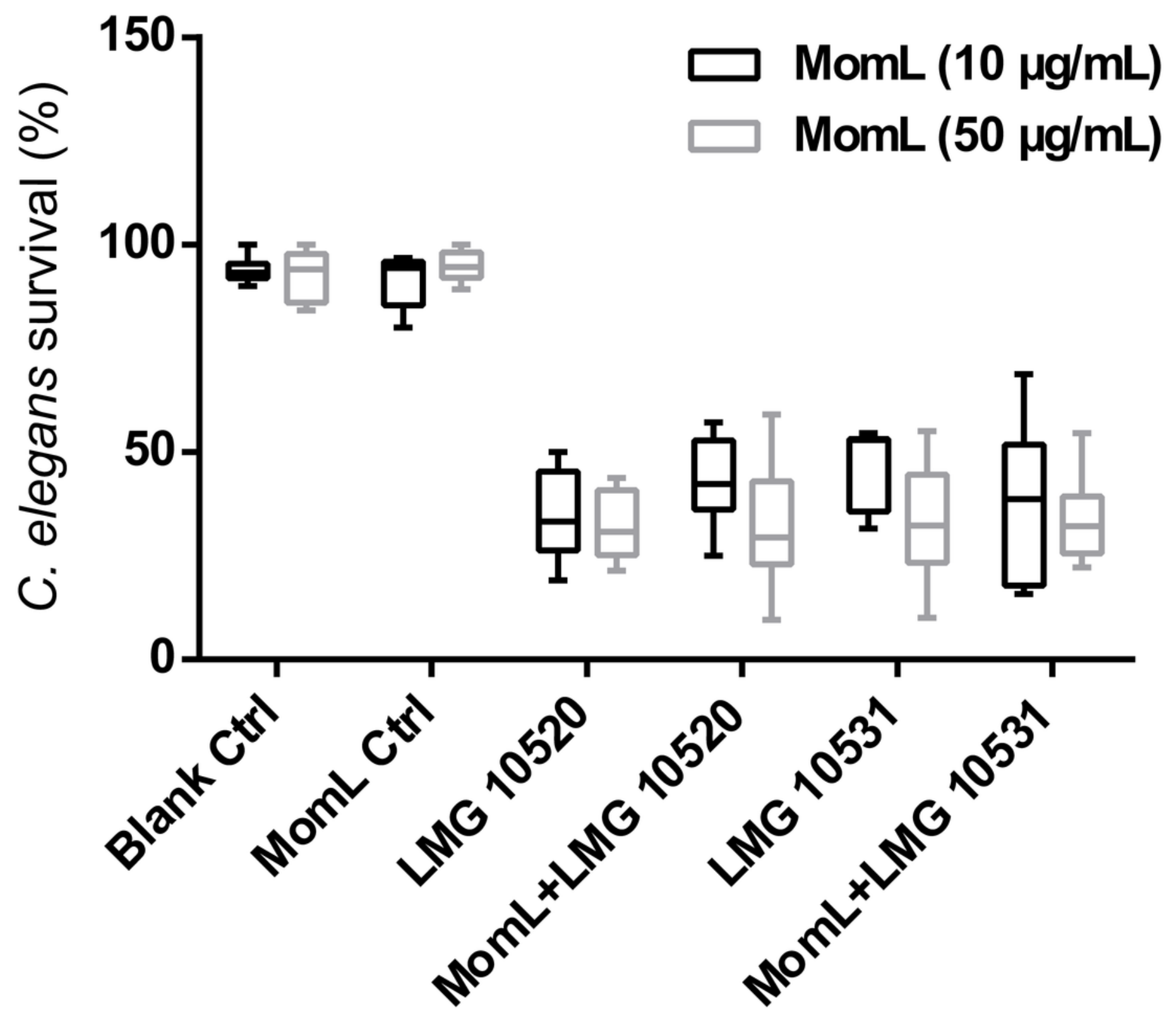

